Departamento de Enfermedades Respiratorias, Facultad de Medicina, Pontificia Universidad Católica de Chile. Santiago, Chile. anternos de Medicina, Facultad de Medicina, Pontificia Universidad Católica de Chile. Santiago, Chile.

Fuente de financiamiento: Proyecto FONDECYT 1080671.

Recibido el 6 de mayo de 2016 , aceptado el 29 de septiembre de 2016.

Correspondencia: Dr. Fernando Saldías Peñafiel Departamento de Enfermedades Respiratorias. División de Medicina-Facultad de Medicina, Pontificia Universidad Católica de Chile.

Marcoleta 350, Santiago, Chile. Teléfonos: (562) 26331541 (562) 23543242 Fax: (562) 26335255 fsaldias@med.puc.cl

\section{Predictores clínicos y funcionales del riesgo de cáncer pulmonar en el seguimiento de una cohorte de adultos fumadores}

\author{
FERNANDO SALDÍAS PEÑAFIEL, JOSÉ MANUEL ELOLA ARÁNGUIZa, \\ JAVIER URIBE MONASTERIO ${ }^{a}$, ARTURO MORALES SOTO, \\ ORLANDO DÍAZ PATIÑO
}

\section{Risk factors for the development of lung cancer in a cohort of adult smokers}

Background: Identifying risk factors for lung cancer in the population could improve the cost-effectiveness of early detection programs using thoracic computed tomography (CT). Aim: To examine the risk factors of lung cancer in a cohort of adult smokers. Patients and Methods: An annual clinical and respiratory functional assessment, chest computed tomography for three years and clinical follow up for five years was carried out in 270 patients aged $65 \pm 9$ years, 55\% males, active or former smokers of 10 or more pack-years. Results: Thirty seven percent of patients were active smokers, consuming $37 \pm 26$ packs/year, $85 \%$ had comorbidities, especially chronic obstructive pulmonary disease (COPD) (66\%), hypertension (48\%), diabetes (22\%) and dyslipidemia (42\%). Thirteen percent of patients had family history of lung cancer. Twenty-one cases of lung cancer were detected in the five years follow up, especially squamous cell carcinoma and adenocarcinoma. In the univariate analysis, the main risk factors for lung cancer identified were an age older than 60 years, history of COPD, family history of lung cancer, active smoking, tobacco consumption more than 30 pack/ year and lung hyperinflation. In multivariate analysis, the three independent risk factors for lung cancer were a family history of lung cancer, active smoking and the number of packs per year of tobacco consumption. Conclusions: The identification of risk groups probably will improve the performance of programs for early detection of lung cancer.

(Rev Med Chile 2016; 144: 1382-1390)

Key words: Early Detective of Cancer; Early Diagnosis; Lung Cancer; Risk Factors; Tobacco Smoking.
$\mathrm{E}$ 1 cáncer pulmonar es la neoplasia con mayor letalidad en el mundo, está relacionado con el consumo de tabaco y otros factores de riesgo de la población. La mayoría de los casos son pesquisados en etapas avanzadas de la enfermedad, lo cual limita las alternativas de tratamiento y explica su elevada letalidad (15\% de sobrevida a 5 años $)^{1,2}$. Es la principal causa de muerte por neoplasia en el mundo, y fallecieron alrededor de 1,6 millones de individuos por esta afección en 2012, lo cual corresponde a alrededor del 20\% de las muertes por cáncer ${ }^{1}$. En Estados Unidos, es la principal causa de muerte por cáncer en ambos sexos, observándose un quiebre en las curvas de incidencia vinculadas a disminución en el consumo de tabaco de la población ${ }^{3}$. En Chile, es la 
segunda causa de muerte por cáncer después del cáncer gástrico, y fallecieron 2.848 personas por esta neoplasia en 2012, correspondiendo a una tasa de mortalidad de 16,4 por 100.000 habitantes, predominando en varones mayores de 50 años $(19,5 \text { casos por } 100.000)^{4}$.

La mayoría de los casos están relacionados con el consumo de tabaco de la población, siendo mayor el riesgo en sujetos fumadores o ex-fumadores recientes (menos de 15 años de abstención), en grandes fumadores (más de 20 paq/año), inicio precoz del hábito tabáquico y en portadores de enfermedad pulmonar obstructiva crónica $(\text { EPOC })^{5-11}$. Otros factores de riesgo de cáncer pulmonar son la exposición a asbesto, arsénico y radón, vinculados a la exposición laboral y domiciliaria ${ }^{1-3}$. En Chile, se estima que fuma alrededor del $40 \%$ de la población mayor de 15 años, es decir, seis millones de individuos, fuman en promedio diez cigarrillos diarios, predominando en varones de estrato socioeconómico medio-alto, ocasionando alrededor de 16 mil muertes cada año ${ }^{12}$. El riesgo de cáncer pulmonar disminuye al dejar de fumar, lo cual está relacionado a la magnitud del consumo de tabaco y al tiempo de suspensión del hábito ${ }^{5-11}$.

Los programas de detección precoz de cáncer pulmonar en población de riesgo, empleando tomografía computarizada de tórax (TAC) con multidetector han permitido obtener imágenes volumétricas de alta resolución, lo que ha permitido detectar el cáncer pulmonar en etapas más precoces de la enfermedad ${ }^{13}$. El National Lung Screening Trial, estudio de tamizaje patrocinado por el National Cancer Institute de Estados Unidos, demostró una reducción del $20 \%$ en el riesgo de morir por cáncer de pulmón en sujetos entre 55 y 74 años fumadores o ex-fumadores de al menos 30 paq/año que fueron evaluados con TAC de tórax comparado con la radiografía de tórax convencional ${ }^{14}$. Por este motivo, se está evaluando los principales grupos de riesgo que se beneficiarían de este estudio de tamizaje $e^{8-11,15-20}$, considerando los costos elevados del examen, los riesgos de la radiación y el alto porcentaje de nódulos inespecíficos benignos detectados en el seguimiento a mediano o largo plazo ${ }^{13,14,21-23}$. El propósito de este estudio es identificar los principales predictores clínicos y funcionales del riesgo de cáncer de pulmón en una cohorte de seguimiento de sujetos mayores de 50 años fumadores.

\section{Pacientes y Métodos}

Estudio clínico descriptivo prospectivo de seguimiento a seis años (enero de 2009 a diciembre de 2015), se enrolaron 270 pacientes mayores de 50 años fumadores o ex-fumadores, quienes aceptaron participar en el estudio clínico firmando un acta de consentimiento informado aprobada por el Comité de Ética en Investigación de la institución. Los criterios de inclusión fueron: adultos mayores de 50 años de ambos sexos, con historia de tabaquismo de 10 o más paquetes año, ser fumador activo o haber dejado de fumar en los últimos treinta años y no tener alguna enfermedad que les impidiera realizar la evaluación funcional respiratoria o que por su gravedad no pudieran completar el período de seguimiento. Se excluyó a los pacientes con historia de asma bronquial, bronquiectasias, fibrosis pulmonar, secuelas de tuberculosis, enfermedad neoplásica conocida, inmunodeficiencia, comorbilidades descompensadas, resección pulmonar, historia de hemoptisis, dolor torácico o baja de peso mayor de $8 \mathrm{~kg}$ en los últimos doce meses de causa desconocida.

\section{Evaluación clínica}

En todos los pacientes incorporados en el estudio se consignó la edad, género, ocupación, consumo de tabaco (expresado en paquetes/ año), condición de fumador activo o ex-fumador, tiempo de suspensión del tabaquismo, comorbilidades, uso de medicamentos, antecedentes personal y familiar de neoplasia, peso, talla e índice de masa corporal (IMC), se evaluó la magnitud de la disnea utilizando la escala de disnea modificada del Medical Research Council del Reino $\mathrm{Unido}^{24}$ (mMRC) y en los pacientes con EPOC se consignó el número de exacerbaciones en los últimos 12 meses, de acuerdo a la definición de Burge y Wedzicha ${ }^{25}$.

\section{Evaluación funcional respiratoria}

1) Se realizó una espirometría con un equipo Sensor Medics 2200 antes y después de la administración de $200 \mu \mathrm{g}$ de salbutamol, siguiendo las normas de las Sociedades Americana de Tórax (ATS) y Europea de Enfermedades Respiratorias (ERS) ${ }^{26}$, empleando los valores de referencia de Hankinson y $\operatorname{cols}^{27}$; 2) Se midió los volúmenes pulmonares (capacidad pulmonar total [CPT], capacidad residual funcional $[\mathrm{CRF}]$, volumen residual $[\mathrm{VR}] \mathrm{y}$ 
capacidad inspiratoria [CI]) en un pletismógrafo corporal Vmax Autobox V62J de BD Medical Supplies, siguiendo las recomendaciones de la ATS/ERS ${ }^{28}$; 3) Se midió la capacidad de difusión de monóxido de carbono $\left(\mathrm{DL}_{\mathrm{CO}}\right)$ siguiendo las recomendaciones de la ATS/ERS ${ }^{9}$, empleando los valores de referencia de Cotes y cols ${ }^{30}$; 4) Se efectuó una prueba de caminata en 6 minutos de acuerdo a las normas de la $\mathrm{ATS}^{31}$. Durante la caminata se registró la $\mathrm{SpO}_{2}$ y frecuencia cardíaca con oxímetro de pulso (Nonin Medical Inc., Plymouth, Mn., EE. UU.). La disnea y fatigabilidad de las piernas se evaluaron empleando la escala psicofísica de Borg $^{32}$. En esta prueba se emplearon los valores de referencia de Troosters y cols ${ }^{33}$; 5) Se calculó el índice de gravedad multidimensional $\mathrm{BODE}^{34}$, que incluye el IMC, $\mathrm{VEF}_{1}$, magnitud de la disnea y distancia recorrida en 6 minutos.

\section{Evaluación clínica en el seguimiento a cinco años}

Se realizó una TAC helicoidal de tórax convencional en todos los pacientes al ser enrolados en el estudio y luego en el seguimiento a los 12 y 24 meses. Dos radiólogos involucrados en el estudio examinaron las imágenes en forma independiente e informaron los hallazgos significativos: pesquisa de nódulos y masas (número, tamaño, localización, textura (sólido, en vidrio esmerilado), características de los bordes, presencia de calcificaciones), adenopatías hiliares y mediastínicas mayores de $1 \mathrm{~cm}$, presencia de opacidades intersticiales o alveolares, derrame pleural o engrosamiento pleural. El examen tomográfico se realizó con el paciente en inspiración profunda, con un equipo multidetector de 64 canales (Somatom Sensation 64, Siemens Healthcare, Erlangen, Alemania), que fue calibrado diariamente para la densidad tomográfica del aire y cada tres meses para el agua. El protocolo de adquisición de imágenes fue: $120 \mathrm{kVp}, 200 \mathrm{mAs}$ y tiempo de rotación de 0,33 segundos. Las imágenes fueron reconstruidas usando un algoritmo de $1 \mathrm{~mm}$ de grosor de corte y $0,7 \mathrm{~mm}$ de intervalo.

El examen tomográfico inicial o de seguimiento fue considerado positivo si se pesquisaron nódulos no calcificados de más de $3 \mathrm{~mm}$ de diámetro, masas en el parénquima pulmonar, o hubo crecimiento de los nódulos en el seguimiento a los 12 o 24 meses. En los nódulos inespecíficos inferiores a $5 \mathrm{~mm}$ de diámetro se sugirió seguimiento tomográfico durante dos años, en los nódulos mayores de $10 \mathrm{~mm}$ o si se objetivó crecimiento del nódulo en el seguimiento se recomendó estudio invasivo (biopsia percutánea bajo TAC, transbronquial o quirúrgica $)^{35}$. En los nódulos de tamaño intermedio se recomendó realizar seguimiento tomográfico o estudio invasivo de acuerdo a los factores de riesgo del paciente y su reserva cardiopulmonar. En el seguimiento a 60 meses, se consignaron las neoplasias broncogénicas pesquisadas mediante tomografía, su variedad histológica, estadio clínico y tratamiento.

\section{Análisis estadístico}

Los resultados son expresados como valores promedio \pm desviación estándar para las variables medidas en escala numérica y en porcentaje para las medidas en escala nominal. Las variables cualitativas fueron comparadas mediante la prueba de $\chi^{2}$ o test exacto de Fisher y las variables continuas con la prueba t de Student. Las variables clínicas y funcionales asociadas al riesgo de cáncer pulmonar fueron sometidas a análisis univariado y multivariado en un modelo de regresión logística (modalidad stepwise) que permite el control simultáneo de múltiples factores. De este modo, los parámetros que no agregaron valor predictivo no fueron retenidos en el modelo. Se calcularon las razones de probabilidad (odds ratio) e intervalos de confianza (IC) para 95\%. Las diferencias entre las variables fueron consideradas significativas con un valor de $\mathrm{p}<0,05$.

\section{Resultados}

Se enrolaron 270 sujetos fumadores en la cohorte de seguimiento a cinco años, la edad promedio era 65,2 $\pm 9,1$ años (rango: $50-80$ ), 55\% sexo masculino, un tercio fumadores activos, consumo de tabaco promedio de $37 \pm 26$ paq/año (rango: 10-150), 85\% tenía comorbilidades, especialmente cardiovasculares, respiratorias y metabólicas crónicas, la mitad referían tos y expectoración crónica (Tabla 1). Dos tercios de los pacientes tenían EPOC, $37 \%$ eran fumadores activos, un tercio en etapas III y IV de GOLD, 5\% eran usuarios de oxígeno domiciliario y $57 \%$ había presentado una o más exacerbaciones de la EPOC en los últimos 12 meses. El 13\% de los pacientes tenía el antecedente de cáncer pulmonar en la familia y se pesquisaron 21 pacientes con cáncer pulmonar $(7,8 \%)$ en el 
Tabla 1. Características clínicas y funcionales de la cohorte de sujetos fumadores

\begin{tabular}{|c|c|c|}
\hline Características & $\mathbf{N}(\mathbf{X} \pm \mathbf{D E})$ & \% (Rango) \\
\hline N & 270 & 100 \\
\hline Edad (años) & $65,2 \pm 9,1$ & (R: 50-80) \\
\hline Género M-F & $149-121$ & $55,2-44,8$ \\
\hline Exfumador-Fumador activo & $171-99$ & $63-37$ \\
\hline Consumo de tabaco (paq/año) & $37,4 \pm 26,2$ & (R: 10-150) \\
\hline Tiempo de suspensión tabaco (años) & $6,8 \pm 9,7$ & (R: 0-30) \\
\hline Antecedente familiar cáncer pulmón & $26 / 204$ & 12,7 \\
\hline Tos y expectoración crónica & 137 & 50,7 \\
\hline Disnea (mMRC $\geq 2$ ) & 101 & 37,4 \\
\hline IMC $\left(\mathrm{Kg} / \mathrm{m}^{2}\right)$ & $26,8 \pm 4,2$ & (R: 16-39,8) \\
\hline Comorbilidades & $229 / 270$ & 84,8 \\
\hline Cardiopatía coronaria & 19 & 7,0 \\
\hline Hipertensión arterial & 130 & 48,1 \\
\hline EPOC & 179 & 66,3 \\
\hline Diabetes mellitus & 59 & 21,9 \\
\hline Dislipidemia & 112 & 41,5 \\
\hline Reflujo gastroesofágico & 82 & 30,4 \\
\hline Obesidad & 57 & 21,1 \\
\hline Depresión & 35 & 13,0 \\
\hline CVF (mL) & $3.380 \pm 980$ & (101 $\pm 20 \%$ teórico) \\
\hline $\operatorname{VEF}_{1}(\mathrm{~mL})$ & $1.970 \pm 860$ & (76 $\pm 29 \%$ teórico) \\
\hline $\mathrm{VEF}_{1} / \mathrm{CVF}(\%)$ & $57,7 \pm 17,9$ & (R: 16-92) \\
\hline $\mathrm{DLCO}(\mathrm{mL} / \mathrm{min} / \mathrm{mmHg})$ & $17,3 \pm 6,4$ & (77 $\pm 23 \%$ teórico) \\
\hline DLCO/VA (mL/min/mmHg/L) & $3,8 \pm 1,0$ & (93 $\pm 24 \%$ teórico) \\
\hline Capacidad pulmonar total (mL) & $6.220 \pm 1.470$ & (114 $\pm 18 \%$ teórico) \\
\hline Capacidad inspiratoria (mL) & $2.530 \pm 790$ & (96 $\pm 23 \%$ teórico) \\
\hline Volumen residual (mL) & $2.680 \pm 1.040$ & (125 $\pm 46 \%$ teórico) \\
\hline Distancia recorrida 6 min (m) & $501 \pm 105$ & (85 $\pm 16 \%$ teórico) \\
\hline
\end{tabular}

Nota: M: Masculino, F: Femenino, IMC: Índice de masa corporal, CVF: Capacidad vital forzada, VEF, Volumen espiratorio forzado del primer segundo, DLCO: Capacidad de difusión de monóxido de carbono, VA: Volumen alveolar, EPOC: Enfermedad Pulmonar Obstructiva Crónica.

seguimiento a cinco años, $52 \%$ correspondieron a carcinoma escamoso y $48 \%$ a adenocarcinoma. La mitad de los pacientes fueron detectados en estadio I ( 11 casos), 3 pacientes en estadio II y III, y 4 pacientes en estadio IV.

Los principales factores de riesgo de cáncer pulmonar identificados en esta cohorte fueron: edad mayor de 60 años (OR: 4,33; IC 95\% 1,0119,1), antecedente de EPOC (OR: 24,8; IC 95\% 1,5-414), historia familiar de cáncer pulmonar (OR: 7,33; IC 95\% 2,2-23,9), tabaquismo activo (OR: 2,48; IC 95\% 1,01-6,12), fumador $\geq 30$ paq/ año (OR: 5,47; IC 95\% 1,3-24), $\mathrm{VEF}_{1} \leq 80 \%$ del valor de referencia (OR: 3,1; IC 95\% 1,1-8,8), 
reducción de la $\mathrm{DL}_{\mathrm{CO}}$ y signos de hiperinsuflación pulmonar objetivado por aumento del volumen residual y capacidad residual funcional y disminución de la capacidad inspiratoria en relación a la capacidad pulmonar total (Tablas 2 y 3 ).

En el análisis multivariado, los principales predictores clínicos del riesgo de cáncer pulmonar en esta cohorte de seguimiento de sujetos fumadores fueron: el antecedente familiar de cáncer pulmonar (OR: 12,7; IC 95\% 3,2-49,9; p: 0,001), la intensidad del consumo de tabaco expresado en paquetes/año (OR: 1,03; IC 95\% 1,01-1,05; p: $0,002)$ y el antecedente de tabaquismo activo (OR: 4,1; IC 95\% 1,1-15; p: 0,03) (Tabla 4).

Tabla 2. Características clínicas y funcionales de los pacientes adultos fumadores con y sin cáncer pulmonar

\begin{tabular}{|c|c|c|c|}
\hline Características & Cáncer pulmonar (+) & Cáncer pulmonar (-) & $\mathbf{p}$ \\
\hline $\mathrm{N}$ & 21 & 249 & \\
\hline Edad (años) & $69,7 \pm 8,3$ & $64,8 \pm 9,1$ & 0,02 \\
\hline Sexo (M-F) & $13-8$ & $136-113$ & 0,52 \\
\hline Fumador activo & $12(57,1 \%)$ & $87(34,9 \%)$ & 0,05 \\
\hline Consumo de tabaco (paq/año) & $54,7 \pm 32,9$ & $36,0 \pm 25,1$ & 0,002 \\
\hline Antecedente familiar de cáncer pulmonar & $6 / 13(46,2 \%)$ & $20 / 191(10,5 \%)$ & 0,001 \\
\hline Tos y expectoración crónica & $14(66,7 \%)$ & $123(49,4 \%)$ & 0,13 \\
\hline Disnea (mMRC $\geq 2$ ) & $11(52,4 \%)$ & $90(36,1 \%)$ & 0,14 \\
\hline $\mathrm{IMC}\left(\mathrm{Kg} / \mathrm{m}^{2}\right)$ & $27,0 \pm 3,5$ & $26,8 \pm 4,2$ & 0,85 \\
\hline Comorbilidades & $19(90,5 \%)$ & $210(84,3 \%)$ & 0,45 \\
\hline Hipertensión arterial & $13(61,9 \%)$ & $117(47,0 \%)$ & 0,19 \\
\hline EPOC & $21(100 \%)$ & $158(63,4 \%)$ & 0,001 \\
\hline Diabetes mellitus & $2(9,5 \%)$ & $57(22,9 \%)$ & 0,16 \\
\hline Dislipidemia & $10(47,6 \%)$ & $102(41,0 \%)$ & 0,55 \\
\hline Reflujo gastroesofágico & $11(52,4 \%)$ & $71(28,5 \%)$ & 0,03 \\
\hline Obesidad & $2(9,5 \%)$ & $55(22,1 \%)$ & 0,18 \\
\hline CVF (mL) & $3.240 \pm 1.240$ & $3.390 \pm 960$ & 0,50 \\
\hline $\operatorname{VEF}_{1}(\mathrm{~mL})$ & $1.770 \pm 770$ & $1.980 \pm 870$ & 0,29 \\
\hline $\mathrm{VEF}_{1} / \mathrm{CVF}(\%)$ & $54,5 \pm 11,7$ & $58,0 \pm 18,3$ & 0,39 \\
\hline $\mathrm{DLCO}(\mathrm{mL} / \mathrm{min} / \mathrm{mmHg})$ & $15,0 \pm 6,7$ & $17,5 \pm 6,3$ & 0,13 \\
\hline DLCO/NA (mL/min/mmHg/L) & $3,4 \pm 1,0$ & $3,9 \pm 1,0$ & 0,05 \\
\hline CRF/CPT & $66,0 \pm 8,1$ & $58,2 \pm 11,1$ & 0,05 \\
\hline VR/CPT & $48,9 \pm 8,1$ & $42,4 \pm 11,1$ & 0,10 \\
\hline $\mathrm{Cl} / \mathrm{CPT}$ & $34,0 \pm 8,1$ & $41,7 \pm 11,1$ & 0,05 \\
\hline Distancia recorrida 6 min (m) & $470 \pm 81$ & $503 \pm 106$ & 0,19 \\
\hline
\end{tabular}

Nota: M: Masculino, F: Femenino, IMC: Índice de masa corporal, EPOC: Enfermedad Pulmonar Obstructiva Crónica, CVF: Capacidad vital forzada, $V_{E F}$ : Volumen espiratorio forzado del primer segundo, DLCO: Capacidad de difusión de monóxido de carbono, VA: Volumen alveolar, CPT: Capacidad pulmonar total, CRF: Capacidad residual funcional, VR: Volumen residual, Cl: Capacidad inspiratoria. 
Tabla 3. Predictores clínicos y funcionales de cáncer pulmonar en una cohorte de sujetos fumadores. Análisis univariado

\begin{tabular}{|c|c|c|c|}
\hline Características & Odds ratio & IC $95 \%$ & $\mathbf{p}$ \\
\hline Edad $>60$ años & 4,33 & $1,01-19,1$ & 0,050 \\
\hline Enfermedad Pulmonar Obstructiva Crónica & 24,8 & $1,49-414,6$ & 0,026 \\
\hline Antecedente familiar de cáncer pulmonar & 7,33 & $2,24-23,96$ & 0,001 \\
\hline Fumador activo & 2,48 & $1,01-6,12$ & 0,049 \\
\hline Fumador $\geq 30$ paq/año & 5,47 & $1,25-24,02$ & 0,025 \\
\hline $\operatorname{VEF}_{1}<1,7 \mathrm{~L}$ & 2,71 & $1,08-6,78$ & 0,034 \\
\hline $\mathrm{VEF}_{1} \leq 80 \%$ teórico & 3,10 & $1,10-8,80$ & 0,034 \\
\hline $\mathrm{VEF}_{1} / \mathrm{CVF}<0,7$ & 22,8 & $1,37-381,1$ & 0,030 \\
\hline $\mathrm{DLCO}<15 \mathrm{~mL} / \mathrm{min} / \mathrm{mmHg}$ & 3,31 & $1,12-9,86$ & 0,032 \\
\hline DLCO $<80 \%$ teórico & 2,64 & $0,83-8,43$ & 0,101 \\
\hline $\mathrm{DLCO} / \mathrm{NA} \leq 4,2$ & 9,81 & $1,27-75,62$ & 0,029 \\
\hline$V R>3,0 \mathrm{~L}$ & 4,37 & $1,01-18,93$ & 0,049 \\
\hline $\mathrm{CRF} / \mathrm{CPT}>0,6$ & 4,89 & $1,01-24,87$ & 0,050 \\
\hline $\mathrm{VR} / \mathrm{CPT}>0,5$ & 4,61 & $1,07-19,97$ & 0,042 \\
\hline $\mathrm{Cl} / \mathrm{CPT}<0,42$ & 25,3 & $1,44-445,3$ & 0,027 \\
\hline Distancia recorrida en $6 \mathrm{~min} \leq 515 \mathrm{~m}$ & 7,40 & $1,67-32,90$ & 0,009 \\
\hline
\end{tabular}

Tabla 4. Predictores clínicos y funcionales de cáncer pulmonar en una cohorte de sujetos fumadores. Análisis multivariado

\begin{tabular}{|lccccc|}
\hline Predictores clínicos & Coeficiente & Error estándar & OR & IC 95\% & p \\
\hline Antecedente familiar de cáncer pulmonar & 2,5412 & 0,6993 & 12,69 & $3,22-49,99$ & 0,001 \\
\hline Consumo de tabaco (paq/año) & 0,0328 & 0,0103 & 1,03 & $1,01-1,05$ & 0,002 \\
\hline Fumador activo & 1,4089 & 0,6640 & 4,09 & $1,11-15,03$ & 0,03 \\
\hline
\end{tabular}

\section{Discusión}

Los principales hallazgos del estudio fueron: a) El riesgo de cáncer pulmonar es elevado en sujetos fumadores o ex-fumadores de más de 10 paq/año; b) Las principales variedades histológicas de la neoplasia pulmonar pesquisadas fueron carcinoma escamoso y adenocarcinoma; c) Los principales predictores clínicos asociados al riesgo de cáncer pulmonar identificados fueron la edad avanzada, magnitud del consumo de tabaco expresado en paquetes/año, ser fumador activo y el antecedente familiar de cáncer pulmonar; d) El riesgo de cáncer pulmonar es más elevado en pacientes con enfermedad pulmonar obstructiva crónica, $\mathrm{VEF}_{1}$ y $\mathrm{DL}_{\mathrm{CO}}$ disminuidos y signos de hiperinsuflación pulmonar.

El rendimiento diagnóstico de la tomografía computarizada de tórax de baja dosis en la pesquisa precoz de cáncer pulmonar en población de riesgo ha demostrado ser superior a la radiografía de tórax ${ }^{13,14,21-23}$. Basados en los estudios controlados se estima que se requiere evaluar a 320 pacientes con TAC de tórax para prevenir una muerte por cáncer pulmonar, lo cual es considerado un método de screening de elevado costo y se requiere optimizar su costo-efectividad ${ }^{36}$. Por este motivo, se están evaluando los principales factores de ries- 
Tabla 5. Modelos epidemiológicos de predicción del riesgo de cáncer pulmonar en la población ${ }^{10,15-20}$

\begin{tabular}{|c|c|c|c|c|c|c|c|}
\hline Factores de riesgo & Bach $^{15}$ & Cassidy $^{16}$ & Spitz $^{17}$ & Etzel ${ }^{18}$ & 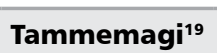 & Tammemagi $^{10}$ & Hoggart ${ }^{20}$ \\
\hline Edad & Sí & Sí & Sí & Sí & Sí & Sí & Sí \\
\hline Sexo & Sí & Sí & Sí & Sí & No & No & No \\
\hline Índice masa corporal & No & No & No & No & Sí & Sí & No \\
\hline Tabaquismo activo & No & No & Sí & Sí & Sí & Sí & Sí \\
\hline Cigarrillos/día & Sí & No & No & No & No & Sí & Sí \\
\hline Duración tabaquismo & $\mathrm{Si}$ & Si & No & No & Si & Sí & Sí \\
\hline Paquetes/año & No & No & Sí & Sí & Sí & No & No \\
\hline Edad inicio tabaco & No & No & No & No & No & No & Sí \\
\hline Suspensión tabaco & Sí & No & Sí & Sí & Sí & Sí & No \\
\hline Cáncer pulmonar en la familia & No & Sí & Sí & No & Sí & Sí & No \\
\hline Exposición a asbesto & Sí & Sí & Sí & No & No & No & No \\
\hline Exposición a polvo & No & No & Sí & Sí & No & No & No \\
\hline EPOC & No & No & No & Sí & Sí & Sí & No \\
\hline Neumonía & No & Sí & No & Sí & No & No & No \\
\hline \multicolumn{8}{|l|}{ Características del modelo } \\
\hline Tipo de estudio & Cohorte & $\begin{array}{l}\text { Caso- } \\
\text { Control }\end{array}$ & $\begin{array}{l}\text { Caso- } \\
\text { Control }\end{array}$ & $\begin{array}{l}\text { Caso- } \\
\text { Control }\end{array}$ & Cohorte & Cohorte & Cohorte \\
\hline Tamaño muestral & 18.172 & 1.736 & 3.852 & 988 & 70.962 & 80.375 & 169.035 \\
\hline Validación interna & Sí & Sí & Sí & Sí & Sí & Sí & No \\
\hline Validación externa & Sí & Sí & Sí & Sí & Sí & Sí & Sí \\
\hline
\end{tabular}

go de cáncer pulmonar en la población y se han diseñado varios modelos predictivos para mejorar el rendimiento de los programas de detección precoz de cáncer pulmonar (Tabla 5) (0,15-20 $^{\text {. }}$

En nuestro estudio, los principales predictores clínicos del riesgo de cáncer de pulmón en sujetos fumadores fueron el antecedente de EPOC y cáncer de pulmón en la familia, ser fumador activo y grandes fumadores. De este modo, el rendimiento del examen sería más elevado en sujetos mayores de 50 años, grandes fumadores (más de 30 paq/ año), ex-fumadores recientes (menos de 15 años de abstención), portadores de enfermedad pulmonar obstructiva crónica y en pacientes con antece-

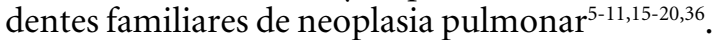

La edad avanzada, la magnitud del consumo de tabaco (expresado en paq/año o la duración y consumo diario de cigarrillos) y el tabaquismo activo están asociados al riesgo de cáncer pulmonar en la población, similar a lo descrito en los modelos predictivos diseñados por Bach y cols. ${ }^{15}$, Cassidy y cols. ${ }^{16}$, Spitz cols. ${ }^{17}$, Tammemagi y cols. ${ }^{19}$, y Hoggart y cols ${ }^{20}$. Por este motivo, la Sociedad Americana de Oncología Clínica ha recomendado evaluar los programas de detección precoz de cáncer pulmonar mediante tomografía en la población de alto riesgo, entre 55 y 74 años, fumadores o ex-fumadores recientes de más de 30 paq/año y con expectativas de vida que justifiquen esta intervención: mejorar la calidad de vida y sobrevida de los enfermos ${ }^{37}$.

La eficacia de los métodos de screening mejora al focalizar los recursos en los grupos de riesgo, sabemos que el $80-90 \%$ de los cánceres de pulmón se presentan en pacientes fumadores, sin embargo, sólo el 10-15\% de los grandes fumadores desarrollarán esta neoplasia ${ }^{1-3}$. Los pacientes con enfermedad pulmonar obstructiva crónica y signos de hiperinsuflación pulmonar, predictores clínicos descritos en nuestro estudio, tienen hasta seis veces más riesgo de cáncer de pulmón entre los pacientes fumadores ${ }^{38,39}$. Se ha planteado que los 
pacientes con enfermedad pulmonar obstructiva crónica y cáncer de pulmón compartirían ciertas características genéticas comunes que favorecerían el desarrollo de estas enfermedades frente a la exposición prolongada a humo de tabaco ${ }^{40,41}$.

La mayoría de los estudios de tamizaje no han demostrado mejoría significativa en la supervivencia' ${ }^{13,21-23}$, excepto el National Lung Screening Trial (NLST) realizado en Estados Unidos que enroló a más de cincuenta mil individuos entre 55 y 74 años, fumadores o ex-fumadores de al menos 30 paq/año y demostró una reducción del 20\% (IC 95\% 6,8-26,7\%, p: 0,004) en la mortalidad asociada a la neoplasia en el seguimiento a mediano plazo $^{14}$. Los costos elevados del examen, los riesgos asociados a la radiación y el elevado porcentaje de nódulos pulmonares inespecíficos de etiología benigna detectados en las pruebas de tamizaje han determinado que la mayoría de los sistemas de salud no recomienden su uso extensivo y de rutina en las poblaciones de riesgo ${ }^{13,42,43}$. Los modelos predictivos $^{10,15-20}$ descritos en la Tabla 5 tienen como objetivo identificar a los principales grupos de riesgo de cáncer de pulmón en la población, en quienes se deberían focalizar los recursos sanitarios para mejorar la costo-efectividad y relación riesgo-beneficio de los estudios de tamizaje con tomografía computarizada de tórax.

En nuestro estudio, similar a lo descrito en los principales modelos predictivos poblacionales ${ }^{15-20}$, los principales factores de riesgo de cáncer pulmonar identificados en sujetos fumadores fueron la edad avanzada, el antecedente de enfermedad pulmonar obstructiva crónica y cáncer de pulmón en la familia, ser fumadores activos y grandes fumadores cuantificado mediante la cantidad de cigarrillos diarios consumidos y la duración de la exposición a tabaco o en paquetes/año.

\section{Referencias}

1. World Health Organization. International Agency for Research on Cancer. GLOBOCAN 2012: Estimated Cancer Incidence, Mortality and Prevalence Worldwide in 2012. Lung Cancer. Accessed December 28, 2015.

2. Centers for Disease Control and Prevention. National Center for Health Statistics. CDC WONDER On-line Database, compiled from Compressed Mortality File 1999-2012 Series 20 No. 2R, 2014.

3. American Cancer Society. Cancer Facts \& Figure 2013. Atlanta, GA: American Cancer Soc; 2013.
4. Ministerio de Salud. Gobierno de Chile. Departamento de Estadísticas e Información de Salud. Serie de mortalidad por causa 2000-2012. http://deis.minsal.cl/vitales/ Mortalidad_causa/Chile.htm

5. Strauss GM. Screening for lung cancer: an evidence-based synthesis. Surg Oncol Clin N Am 1999; 8: 747-74.

6. Osann KE. Lung cancer in women: the importance of smoking, family history of cancer, and medical history of respiratory disease. Cancer Res 1991; 51: 4893-7.

7. Burns DM. Primary prevention, smoking, and smoking cessation: implications for future trends in lung cancer prevention. Cancer 2000; 89 (11 Suppl): 2506-9.

8. Halpern MT, Gillespie BW, Warner KE. Patterns of absolute risk of lung cancer mortality in former smokers. J Natl Cancer Inst 1993; 85: 457-64.

9. Raji OY, Duffy SW, Agbaje OF, Baker SG, Christiani DC, Cassidy A, et al. Predictive accuracy of the Liverpool Lung Project risk model for stratifying patients for computed tomography screening for lung cancer: a case-control and cohort validation study. Ann Intern Med 2012; 157: 242-50.

10. Tammemagi MC, Katki HA, Hocking WG, Church TR, Caporaso N, Kvale PA, et al. Selection criteria for lung-cancer screening. N Engl J Med 2013; 368: 728-36.

11. Kovalchik SA, Tammemagi M, Berg CD, Caporaso NE, Riley TL, Korch M, et al. Targeting of low-dose CT screening according to the risk of lung-cancer death. N Engl J Med 2013; 369: 245-54.

12. Ministerio de Salud. Exposición a tabaco. Encuesta Nacional de Salud 2009-2010, Chile. Págs. 152-88.

13. Bach PB, Mirkin JN, Oliver TK, Azzoli CG, Berry DA, Brawley OW, et al. Benefits and harms of CT screening for lung cancer: a systematic review. JAMA 2012; 307: 2418-29.

14. National Lung Screening Trial Research Team, Aberle DR, Adams AM, Berg CD, Black WC, Clapp JD, Fagerstrom RM, et al. Reduced lung-cancer mortality with low-dose computed tomographic screening. N Engl J Med 2011; 365: 395-409.

15. Bach PB, Kattan MW, Thornquist MD, Kris MG, Tate RC, Barnett MJ, et al. Variations in lung cancer risk among smokers. J Natl Cancer Inst 2003; 95: 470-8.

16. Cassidy A, Myles JP, van Tongeren M, Page RD, Liloglou T, Duffy SW, et al. The LLP risk model: an individual risk prediction model for lung cancer. Br J Cancer 2008; 98: 270-6.

17. Spitz MR, Hong WK, Amos CI, Wu X, Schabath $\mathrm{MB}$, Dong Q, et al. A risk model for prediction of lung cancer. J Natl Cancer Inst 2007; 99: 715-26.

18. Etzel CJ, Kachroo S, Liu M, D’Amelio A, Dong Q, Cote $\mathrm{ML}$, et al. Development and validation of a lung cancer 
risk prediction model for African-Americans. Cancer Prev Res (Phila) 2008; 1: 255-65.

19. Tammemagi CM, Pinsky PF, Caporaso NE, Kvale PA, Hocking WG, Church TR, et al. Lung cancer risk prediction: prostate, lung, colorectal and ovarian cancer screening trial models and validation. J Natl Cancer Inst 2011; 103: 1058-68.

20. Hoggart C, Brennan P, Tjonneland A, Vogel U, Overvad K, Østergaard JN, et al. A risk model for lung cancer incidence. Cancer Prev Res (Phila) 2012; 5: 834-46.

21. Infante M, Cavuto S, Lutman FR, Brambilla G, Chiesa G, Ceresoli G, et al; DANTE Study Group. A randomized study of lung cancer screening with spiral computed tomography: three-year results from the DANTE trial. Am J Respir Crit Care Med 2009; 180: 445-53.

22. Saghir Z, Dirksen A, Ashraf H, Bach KS, Brodersen J, Clementsen PF, et al. CT screening for lung cancer brings forward early disease. The randomized Danish Lung Cancer Screening Trial: status after five annual screening rounds with low-dose CT. Thorax 2012; 67: 296-301.

23. Pastorino U, Rossi M, Rosato V, Marchiano A, Sverzellati N, Morosi C, et al. Annual or biennial CT screening versus observation in heavy smokers: 5-year results of the MILD trial. Eur J Cancer Prev 2012; 21: 308-15.

24. Mahler DA, Wells CK. Evaluation of clinical methods for rating dyspnea. Chest 1988; 93: 580-6.

25. Burge S, Wedzicha JA. COPD exacerbations: definitions and classifications. Eur Respir J 2003; 41 (Suppl): 46S-53S.

26. Miller MR, Hankinson J, Brusasco V, Burgos F, Casaburi $\mathrm{R}$, Coates A, et al. Standardisation of spirometry. Eur Respir J 2005; 26: 319-38.

27. Hankinson JL, Odencrantz JR, Fedan KB. Spirometric reference values from a sample of the general U.S. population. Am J Respir Crit Care Med 1999; 159: 179-87.

28. Wanger J, Clausen JL, Coates A, Pedersen OF, Brusasco $\mathrm{V}$, Burgos F, et al. Standardisation of the measurement of lung volumes. Eur Respir J 2005; 26: 511-22.

29. MacIntyre N, Crapo RO, Viegi G, Johnson DC, van der Grinten CP, Brusasco V, et al. Standardisation of the single-breath determination of carbon monoxide uptake in the lung. Eur Respir J 2005; 26: 720-35.

30. Cotes JE, Chinn DJ, Quanjer PH, Roca J, Yernault JC. Standardization of the measurement of transfer factor (diffusing capacity). Report Working Party Standardization of Lung Function Tests, European Community for Steel and Coal. Official Statement of the European Respiratory Society. Eur Respir J 1993; 16 (Suppl): 4152.
31. American Thoracic Society Statement. Guidelines for the six-minute walk test. Am J Respir Crit Care Med 2002; 166: 111-7.

32. Troosters T, Gosselink S, Decramer M. Six minute walking distance in healthy elderly subjects. Eur Respir J 1999; 14: 270-4.

33. Borg GA. Psychophysical basis of perceived exertion. Med Sci Sports Exerc 1982; 14: 377-81.

34. Celli BR, Cote CG, Marín JM, Casanova C, Montes de Oca M, Méndez RA, et al. The body-mass index, airflow obstruction, dyspnea and exercise capacity index in chronic obstructive pulmonary disease. N Engl J Med 2004; 350: 1005-12.

35. Henschke CI, Yankelevitz DF, Libby DM, McCauley D, Pasmantier M, Altorki NK, et al. Early lung cancer action project: annual screening using single-slice helical CT. Ann N Y AcadSci 2001; 952: 124-34.

36. Nitadori J, Inoue $\mathrm{M}$, Iwasaki $\mathrm{M}$, Otani $\mathrm{T}$, Sasazuki $S$, Nagai K, et al. Association between lung cancer incidence and family history of lung cancer: data from a large-scale population-based cohort study, the JPHC study. Chest 2006; 130: 968-75.

37. Wender R, Fontham ET, Barrera E Jr, Colditz GA, Church TR, Ettinger DS, et al. American Cancer Society lung cancer screening guidelines. CA Cancer J Clin 2013; 63: 107-17.

38. Punturieri A, Szabo E, Croxton TL, Shapiro SD, Dubinett SM. Lung cancer and chronic obstructive pulmonary disease: needs and opportunities for integrated research. J Natl Cancer Inst 2009; 101: 554-9.

39. Young RP, Hopkins RJ, Christmas T, Black PN, Metcalf $\mathrm{P}$, Gamble GD. COPD prevalence is increased in lung cancer, independent of age, sex and smoking history. Eur Respir J 2009; 34: 380-6.

40. Adcock IM, Caramori G, Barnes PJ. Chronic obstructive pulmonary disease and lung cancer: New molecular insights. Respiration 2011; 81: 265-84.

41. Young RP, Hopkins RJ, Hay BA, Whittington CF, Epton MJ, Gamble GD. FAM13A locus in COPD is independently associated with lung cancer: Evidence of a molecular genetic link between COPD and lung cancer. Appl Clin Genet 2010; 4: 1-10.

42. Humphrey LL, Deffebach M, Pappas M, Baumann C, Artis K, Mitchell JP, et al. Screening for lung cancer with low-dose computed tomography: A systematic review to update the U.S. preventive services task force recommendation. Ann Intern Med 2013; 159: 411-20.

43. Manser R, Lethaby A, Irving LB, Stone C, Byrnes G, Abramson MJ, et al. Screening for lung cancer. Cochrane Database Syst Rev 2013 Jun 21; 6: CD001991. 